TRANSACTIONS OF THE

AMERICAN MATHEMATICAL SOCIETY

Volume 364, Number 3, March 2012, Pages 1145-1162

S 0002-9947(2011)05512-1

Article electronically published on October 24, 2011

\title{
NON-INTEGRATED DEFECT RELATION FOR MEROMORPHIC MAPS OF COMPLETE KÄHLER MANIFOLDS INTO $\mathbb{P}^{n}(\mathbb{C})$ INTERSECTING HYPERSURFACES
}

\author{
MIN RU AND SURAIZOU SOGOME
}

\begin{abstract}
In this paper, we establish a non-integrated defect relation for a meromorphic map of a complete Kähler manifold whose universal covering is biholomorphic to a ball in $\mathbb{C}^{m}$ into $\mathbb{P}^{n}(\mathbb{C})$ intersecting hypersurfaces in general position, as well as an application to the Gauss map of a closed regular submanifold of $\mathbb{C}^{m}$. The result provides a complement to the recent result of $\mathrm{Ru}(2004)$ on a defect relation for meromorphic mappings from $\mathbb{C}^{m}$ into $\mathbb{P}^{n}(\mathbb{C})$ intersecting hypersurfaces in general position.
\end{abstract}

\section{INTRODUCTION}

Relating to the study of the value distribution of the Gauss maps of complete minimal surfaces in $\mathbb{R}^{m}, \mathrm{H}$. Fujimoto (see 4]) introduced the notion of the nonintegrated defect for a holomorphic map of an open Riemann surface $M$ into $\mathbb{P}^{n}(\mathbb{C})$ and obtained some results analogous to the classical defect relation. In 5, he generalized these results to the case of a meromorphic map of a complete Kähler manifold $M$ whose universal covering is biholomorphic to a ball in $\mathbb{C}^{m}$ into $\mathbb{P}^{n_{1}}(\mathbb{C}) \times$ $\cdots \times \mathbb{P}^{n_{k}}(\mathbb{C})$ and the meromorphic map satisfies a certain growth condition (see the condition in Theorem 1.1).

Let $M$ be an $m$-dimensional complex Kähler manifold. Let $f$ be a meromorphic map of $M$ into $\mathbb{P}^{n}(\mathbb{C}), \mu_{0}$ be a positive integer and $D$ be a hypersurface in $\mathbb{P}^{n}(\mathbb{C})$ of degree $d$ with $f(M) \not \subset D$. We denote the intersection multiplicity of the image of $f$ and $D$ at $f(p)$ by $\nu^{f}(D)(p)$ and the pull-back of the normalized Fubini-Study metric form $\Omega$ on $\mathbb{P}^{n}(\mathbb{C})$ by $\Omega_{f}$. The non-integrated defect of $f$ with respect to $D$ cut by $\mu_{0}$ is defined by

$$
\delta_{\mu_{0}}^{f}(D):=1-\inf \{\eta \geq 0: \eta \text { satisfies condition }(\star)\} .
$$

Here, the condition $(\star)$ means that there exists a bounded non-negative continuous function $h$ on $M$ with zeros of order not less than $\min \left(\nu^{f}(D), \mu_{0}\right)$ such that

$$
d \eta \Omega_{f}+\frac{\sqrt{-1}}{2 \pi} \partial \bar{\partial} \log h^{2} \geq\left[\min \left(\nu^{f}(D), \mu_{0}\right)\right],
$$

Received by the editors January 26, 2010.

2010 Mathematics Subject Classification. Primary 32H30; Secondary 53A10.

Key words and phrases. Meromorphic mappings, Gauss map of minimal surfaces, defect relation, Nevanlinna theory.

The first author was supported in part by NSA under grant number H98230-09-1-0004 and H98230-11-0201.

(C)2011 American Mathematical Society Reverts to public domain 28 years from publication 
where $d$ is the degree of $D$ and we mean by $[\nu]$ the $(1,1)$-current associated with a divisor $\nu$. Note that the condition $(\star)$ also means that, for each holomorphic function $\phi(\not \equiv 0)$ on an open subset $U$ of $M$ with $\nu_{\phi}=\min \left(\nu^{f}(D), \mu_{0}\right)$ outside an analytic set of codimension $\geq 2$, the function $u:=\log \left(h^{2}\|f\|^{2 d \eta} /|\phi|^{2}\right)$ is continuous and plurisubharmonic on $U$, where $\|f\|^{2}=\left|f_{0}\right|^{2}+\cdots+\left|f_{n}\right|^{2}$, and $f=\left[f_{0}: \cdots: f_{n}\right]$ is a (local) reduced representation of $f$. So, similar to the classical Nevanlinna's defect, we have the following properties.

- $0 \leq \delta_{\mu_{0}}^{f}(D) \leq 1$. To see $\delta_{\mu_{0}}^{f}(D) \geq 0$, take $\eta=1$ and $h=|Q(f)| /\|f\|^{d}$, where $Q$ is the homogeneous polynomial defining $D$.

- If $f(M) \cap D=\emptyset$, then, by taking $\eta=0, h=1$, we have that $\delta_{\mu_{0}}^{f}(D)=1$.

- If $\nu^{f}(D)(p) \geq \mu$ for all $p \in f^{-1}(D)$, with some positive integer $\mu \geq \mu_{0}$, then $\delta_{\mu_{0}}^{f}(D) \geq 1-\mu_{0} / \mu$ by taking $\eta=\mu_{0} / \mu$ and $h=\left(|Q(f)| /\|f\|^{d}\right)^{\mu_{o} / \mu}$.

Let $\omega=\frac{\sqrt{-1}}{2} \sum_{i j} h_{i \bar{j}} d z_{i} \wedge d \bar{z}_{j}$ be the Kähler form of $M$. We define

$$
\operatorname{Ric} \omega=d d^{c} \log \left(\operatorname{det}\left(h_{i j}\right)\right) \text {, }
$$

where $d=\partial+\bar{\partial}$ and $d^{c}=\frac{\sqrt{-1}}{4 \pi}(\bar{\partial}-\partial)$.

We assume the following growth condition for $f$ : there exists a non-zero bounded continuous real-valued function $h$ on $M$ such that $\rho \Omega_{f}+\frac{\sqrt{-1}}{2 \pi} \partial \bar{\partial} \log h^{2} \geq$ Ric $\omega$ for some non-negative constant $\rho$. In this paper, we extend the results in [5] to the case where $f(M)$ intersects with hypersurfaces instead of hyperplanes; namely, we prove the following main theorem.

Theorem 1.1. Let $M$ be an m-dimensional complete Kähler manifold and $f: M \rightarrow$ $\mathbb{P}^{n}(\mathbb{C})$ be a meromorphic map which is algebraically non-degenerate (i.e., its image is not contained in any proper subvariety of $\left.\mathbb{P}^{n}(\mathbb{C})\right)$. Assume that the universal covering of $M$ is biholomorphic to a ball in $\mathbb{C}^{m}$. Let $D_{1}, \ldots, D_{q}$ be hypersurfaces of degree $d_{j}$ in $\mathbb{P}^{n}(\mathbb{C})$, located in general position. Let $d=$ l.c.m. $\left\{d_{1}, \ldots, d_{q}\right\}$ (the least common multiple of $\left.\left\{d_{1}, \ldots, d_{q}\right\}\right)$. Assume that there exists a non-zero bounded continuous real-valued function $h$ on $M$ such that $\rho \Omega_{f}+\frac{\sqrt{-1}}{2 \pi} \partial \bar{\partial} \log h^{2} \geq$ Ric $\omega$ for some non-negative constant $\rho$. Then, for every $\epsilon>0$,

$$
\sum_{j=1}^{q} \delta_{l-1}^{f}\left(D_{j}\right) \leq n+1+\epsilon+\frac{\rho l(l-1)}{d},
$$

where $l \leq 2^{n^{2}+4 n} e^{n} d^{2 n}\left(n I\left(\epsilon^{-1}\right)\right)^{n}$ and $I(x):=\min \{k \in \mathbb{N}: k>x\}$ for a positive real number $x$.

We remark that in the case $M=\mathbb{C}^{m}$, we also have the following statement (see Theorem 4.5 and Corollary 4.6) which is essentially due to Min Ru (see [10] and 9]) without the truncation and to An and Phuong with the truncation (see [1]).

Theorem 1.2. Let $f: \mathbb{C}^{m} \longrightarrow \mathbb{P}^{n}(\mathbb{C})$ be a meromorphic map which is algebraically non-degenerate. Let $D_{1}, \ldots, D_{q}$ be hypersurfaces of degree $d_{j}$ in $\mathbb{P}^{n}(\mathbb{C})$, located in general position. Let $d=$ l.c.m. $\left\{d_{1}, \ldots, d_{q}\right\}$. Then, for every $\epsilon>0$,

$$
\sum_{j=1}^{q} \delta_{l-1}^{f, \star}\left(D_{j}\right) \leq n+1+\epsilon,
$$


where $l \leq 2^{n^{2}+4 n} e^{n} d^{2 n}\left(n I\left(\epsilon^{-1}\right)\right)^{n}, I(x):=\min \{k \in \mathbb{N}: k>x\}$ for a positive real number $x$, and $\delta_{l-1}^{f, \star}(D)$ is the classical Nevanlinna's (truncated) defect of $f$ with respect to $D$.

Note that, from the discussion below, we have that $\delta_{l-1}^{f}(D) \leq \delta_{l-1}^{f, *}(D)$ (see Proposition 2.1). Thus, Theorem 1.1 and Theorem 1.2 are complementing each other.

The main tools of the proof are the following fundamental result of Yau: "Let $M$ be a complete Riemannian manifold of infinite volume and $u$ a non-negative function satisfying $\triangle \log u=0$ almost everywhere. Then $\int_{M} u^{p}=\infty$ for $p>0$," as well as the techniques developed in 10 and 11 of dealing with hypersurfaces in general position. The proof gets down to the construction of such a function $u$ on $M$ which contradicts Yau's result mentioned above if the theorem fails.

\section{Preliminaries}

First of all, throughout the article, we'll use the common letter $K$ to denote a constant, even when it should be replaced by a new constant. Let $h$ be a non-constant holomorphic function on an open domain $G \subset \mathbb{C}^{m}$. For a set $\alpha=\left(\alpha_{1}, \ldots, \alpha_{m}\right)$ of integers $\alpha_{i} \geq 0$, we set $|\alpha|=\alpha_{1}+\cdots+\alpha_{m}$ and $D^{\alpha} h=D_{1}^{\alpha_{1}} \cdots D_{m}^{\alpha_{m}} h$, where $D_{i} h=\left(\partial / \partial z_{i}\right) h$, for $i=1, \ldots, m$. We define $\nu_{h}^{0}: G \rightarrow \mathbb{Z}$ by

$$
\nu_{h}^{0}(z):=\max \left\{k: D^{\alpha} h(z)=0 \text { for all } \alpha \text { with }|\alpha|<k\right\}(z \in G) .
$$

By a divisor on a domain $G$ in $\mathbb{C}^{m}$ we mean a map $\nu$ of $G$ into $\mathbb{Z}$ such that, for each $z_{0} \in G$, there are non-zero holomorphic functions $h$ and $g$ on a connected neighborhood $U(\subset G)$ of $z_{0}$ so that $\nu(z)=\nu_{h}^{0}(z)-\nu_{g}^{0}(z)$ for each $z \in U$ outside an analytic set of dimension $\leq m-2$. Two divisors are regarded as the same if they are identical outside an analytic set of dimension $\leq m-2$.

Take a non-zero meromorphic function $\varphi$ on a domain $G$ in $\mathbb{C}^{m}$. For each $z_{0} \in G$, we choose non-zero holomorphic functions $g$ and $h$ on a neighborhood $U(\subset G)$ of $z_{0}$ such that $\varphi=\frac{g}{h}$ on $U$ and $\operatorname{dim}\left(f^{-1}(0) \cup g^{-1}(0)\right) \leq m-2$. We define $\nu_{\varphi}^{\infty}:=\nu_{h}, \nu_{\varphi}^{a}:=$ $\nu_{g-a h}$ for $a \in \mathbb{C}$ and $\nu_{\varphi}=\nu_{\varphi}^{0}-\nu_{\varphi}^{\infty}$, which are independent of the choices of $h$ and $g$ and so are globally well-defined on $G$. Let $f$ be a meromorphic map of $B\left(R_{0}\right) \subset \mathbb{C}^{m}$ into $\mathbb{P}^{n}(\mathbb{C}), 0<R_{0} \leq \infty$. We take holomorphic functions $f_{0}, f_{1}, \ldots, f_{n}$ such that $I_{f}:=\left\{z \in B\left(R_{0}\right), f_{0}(z)=\cdots=f_{n}(z)=0\right\}$ is of dimension at most $m-2$ and $f(z)=\left[f_{0}(z): \cdots: f_{n}(z)\right]$ on $B\left(R_{0}\right)-I_{f}$ in terms of homogeneous coordinates $\left[w_{0}: \cdots: w_{n}\right]$ on $\mathbb{P}^{n}(\mathbb{C})$. We call such a representation $f(z)=\left[f_{0}(z): \cdots: f_{n}(z)\right]$ a reduced representation of $f$.

For $z=\left(z_{1}, \ldots, z_{m}\right) \in \mathbb{C}^{m}$ we set $\|z\|=\left(\left|z_{1}\right|^{2}+\cdots+\left|z_{m}\right|^{2}\right)^{1 / 2}$ and define $B(r)=$ $\left\{z \in \mathbb{C}^{m}:\|z\|<r\right\}, \quad S(r)=\left\{z \in \mathbb{C}^{m}:\|z\|=r\right\}$ for $0<r \leq+\infty$, where we mean $B(\infty)=\mathbb{C}^{m}$ and $S(\infty)=\emptyset$. Define

$$
\begin{aligned}
\sigma_{m} & :=d^{c} \log \|z\|^{2} \wedge\left(d d^{c} \log \|z\|^{2}\right)^{m-1} \quad \text { on } \quad \mathbb{C}^{m}-\{0\}, \\
v_{l} & :=\left(d d^{c}\|z\|^{2}\right)^{l} \quad \text { for } \quad 1 \leq l \leq m .
\end{aligned}
$$

Let $f(z)=\left[f_{0}(z): \cdots: f_{n}(z)\right]$ be a reduced representation of $f$. Set $\|f\|:=$ $\left(\left|f_{0}\right|^{2}+\cdots+\left|f_{n}\right|^{2}\right)^{1 / 2}$. Then the pull-back of the normalized Fubini-Study metric form $\Omega$ on $\mathbb{P}^{n}(\mathbb{C})$ by $f$ is given by

$$
\Omega_{f}=d d^{c} \log \|f\|^{2} .
$$


Fixing $r_{0}<R_{0}$, the characteristic function of $f$ is defined by

$$
T_{f}\left(r, r_{0}\right)=\int_{r_{0}}^{r} \frac{d t}{t^{2 m-1}} \int_{B(t)} \Omega_{f} \wedge v_{m-1} \quad\left(0<r_{0}<r<R_{0}\right) .
$$

We then have (see [12], pp. 251-255),

$$
T_{f}\left(r, r_{0}\right)=\int_{S(r)} \log \|f\| \sigma_{m}-\int_{S\left(r_{0}\right)} \log \|f\| \sigma_{m} .
$$

Let $\mu_{0}$ be a positive integer or $\infty$ and $\nu$ be a divisor on a domain $B\left(R_{0}\right) \subset \mathbb{C}^{m}$. Set $|\nu|=\overline{\left\{z \in B\left(R_{0}\right): \nu(z) \neq 0\right\}}$. We define the counting function of $\nu$ truncated by $\mu_{0}$ by

$$
N_{\nu}^{\left[\mu_{0}\right]}\left(r_{0}, r\right)=\int_{r_{0}}^{r} \frac{n^{\left[\mu_{0}\right]}(t)}{t} d t
$$

where

$$
\begin{gathered}
n^{\left[\mu_{0}\right]}(t)=\frac{1}{t^{2 m-2}} \int_{|\nu| \cap B(t)} \min \left\{\nu, \mu_{0}\right\} v_{m-1} \quad \text { if } m \geq 2, \\
n^{\left[\mu_{0}\right]}(t)=\sum_{|z| \leq t} \min \left\{\nu(z), \mu_{0}\right\} \quad \text { if } m=1 .
\end{gathered}
$$

Let $D$ be a hypersurface in $\mathbb{P}^{n}(\mathbb{C})$ of degree $d$, and let $Q$ be the homogeneous polynomial defining $D$. We define the divisor $\nu(f, D)(z):=\nu_{Q \circ f}^{0}(z)\left(z \in B\left(R_{0}\right)\right)$ which is rephrased as the intersection multiplicity of the image of $f$ and $D$ at $f(z)$. We define the (truncated) counting function of $D$ for $f$ by, for fixed $r_{0}<R_{0}$,

$$
N_{f}^{\left[\mu_{0}\right]}(r, D):=N_{\nu(f, D)}^{\left[\mu_{0}\right]}\left(r_{0}, r\right) .
$$

By the first main theorem,

$$
N_{f}^{\left[\mu_{0}\right]}(r, D) \leq d T_{f}\left(r, r_{0}\right)+O(1) .
$$

The classical Nevanlinna's defect $\delta_{\mu_{0}}^{f, \star}(D)$ of $f$ with respect to $D$ cut by $\mu_{0}$ is defined by

$$
\delta_{\mu_{0}}^{f, \star}(D)=\limsup _{r \rightarrow R_{0}}\left(1-\frac{N_{f}^{\left[\mu_{0}\right]}(r, D)}{d T_{f}\left(r, r_{0}\right)}\right) .
$$

The relationship between the non-integrated defect and the classical Nevanlinna's defect is given as follows.

Proposition 2.1. If $\lim _{r \rightarrow R_{0}} T_{f}\left(r, r_{0}\right)=\infty$, then

$$
0 \leq \delta_{\mu_{0}}^{f}(D) \leq \delta_{\mu_{0}}^{f, \star}(D) \leq 1,
$$

where $\delta^{\star}$ is the classical Nevanlinna's defect.

Proof. Take $\eta$ satisfying the condition $(\star)$ in the definition of $\delta_{\mu_{0}}^{f}(D)$. The function

$$
v:=d \eta \log \|f\|+\log h-\log |\varphi|
$$


is then plurisubharmonic, where $h$ is bounded and $\varphi$ is holomorphic on $B\left(R_{0}\right)$ with $\nu_{\varphi}=\min \left(\nu^{f}(D), \mu_{0}\right)$ outside an analytic set of codimension $\geq 2$. Therefore,

$$
\begin{aligned}
0 & \leq \int_{S(r)} v \sigma_{m}-\int_{S\left(r_{0}\right)} v \sigma_{m} \\
& =d \eta \int_{S(r)} \log \|f\| \sigma_{m}+\int_{S(r)} \log h \sigma_{m}-\int_{S(r)} \log |\varphi| \sigma_{m}+K \\
& \leq d \eta T_{f}\left(r, r_{0}\right)-N_{f}^{\left[\mu_{0}\right]}(r, D)+K,
\end{aligned}
$$

where $K$ is a constant, because $h$ is bounded from above. This implies that

$$
\frac{N_{f}^{\left[\mu_{0}\right]}(r, D)}{d T_{f}\left(r, r_{0}\right)} \leq \eta+\frac{K}{T_{f}\left(r, r_{0}\right)} .
$$

As $r \rightarrow R_{0}$, we obtain $\delta_{\mu_{0}}^{\star}(D) \geq 1-\eta$. Hence $\delta_{\mu_{0}}^{\star}(D) \geq \delta_{\mu_{0}}^{f}(D)$.

\section{The GENERALIZED WronsKians AND THE LEMMA OF THE LOGARITHMIC DERIVATIVE}

We first recall the following lemma of the logarithmic derivative.

Theorem 3.1 (See [5], Theorem 3.1). Let $\phi$ be a non-zero a meromorphic function on $B\left(R_{0}\right) \subset \mathbb{C}^{m}, 0<R_{0} \leq \infty$, and let $\alpha=\left(\alpha_{1}, \ldots, \alpha_{m}\right) \neq(0, \ldots, 0), 0<r_{0}<R_{0}$, and take positive numbers $p, p^{\prime}$ such that $0<p|\alpha|<p^{\prime}<1$. Then, for $r_{0}<r<$ $R<R_{0}$,

$$
\int_{S(r)}\left|z^{\alpha}\left(D^{\alpha} \phi / \phi\right)(z)\right|^{p} \sigma_{m}(z) \leq K\left(\frac{R^{2 m-1}}{R-r} T_{\phi}\left(R, r_{0}\right)\right)^{p^{\prime}}
$$

where $K$ is a constant not depending on each $r$ and $R$, and $z^{\alpha}:=z_{1}^{\alpha_{1}} \cdots z_{m}^{\alpha_{m}}$ for $z=\left(z_{1}, \ldots, z_{m}\right)$ and $\alpha=\left(\alpha_{1}, \ldots, \alpha_{m}\right)$.

Let $F$ be a meromorphic map of $B\left(R_{0}\right) \subset \mathbb{C}^{m}\left(0<R_{0} \leq \infty\right)$ into $\mathbb{P}^{l-1}(\mathbb{C})$. Take a reduced representation $F=\left[F_{1}: \cdots: F_{l}\right]$. We shall say that $F$ is linearly non-degenerate if $F\left(B\left(R_{0}\right)\right) \not \subset H$ for every hyperplane $H$ in $\mathbb{P}^{l-1}(\mathbb{C})$, or equivalently, $F_{1}, \ldots, F_{l}$ are linearly independent on $\mathbb{C}^{m}$. Take an arbitrary set $\alpha^{i}=\left(\alpha_{i 1}, \ldots, \alpha_{i l}\right), 1 \leq i \leq l$, of non-negative integers. We define the generalized Wronskian of $F$ by

$$
W_{\alpha^{1} \ldots \alpha^{l}}(F)=\operatorname{det}\left(D^{\alpha^{1}} F, \ldots, D^{\alpha^{l}} F\right) .
$$

Proposition 3.2 (See [5], Proposition 4.5). Let $F: B\left(R_{0}\right) \rightarrow \mathbb{P}^{l-1}(\mathbb{C})$ be a linearly non-degenerate meromorphic map. Then there exist $\alpha^{j}=\left(\alpha_{j 1}, \ldots, \alpha_{j l}\right)$ with $\alpha_{j i} \geq$ 0 being integers, $\left|\alpha^{j}\right| \leq l-1$ for $1 \leq j \leq l$, and $\left|\alpha^{1}\right|+\cdots+\left|\alpha^{l}\right| \leq l(l-1) / 2$ such that the generalized Wronskian $W_{\alpha^{1} \ldots \alpha^{l}}(F) \not \equiv 0$.

Take such $\alpha^{j}=\left(\alpha_{j 1}, \ldots, \alpha_{j l}\right), 1 \leq j \leq l$, so that the generalized Wronskian $W_{\alpha^{1} \ldots \alpha^{l}}(F) \not \equiv 0$. Let $L_{1}, \ldots, L_{l}$ be linear forms of $l$ variables and assume that they are linearly independent. Theorem 3.1 implies the following proposition.

Proposition 3.3 (See [5], Proposition 6.1). In the above situation, set $l_{0}=\left|\alpha^{1}\right|+$ $\cdots+\left|\alpha^{l}\right|$ and take $t, p^{\prime}$ with $0<t l_{0}<p^{\prime}<1$. Then, for $0<r_{0}<R_{0}$ there exists a 
positive constant $K$ such that for $r_{0}<r<R<R_{0}$,

$$
\int_{S(r)}\left|z^{\alpha^{1}+\cdots+\alpha^{l}} \frac{W_{\alpha^{1} \ldots \alpha^{l}}(F)}{L_{1}(F) \cdots L_{l}(F)}\right|^{t} \sigma_{m} \leq K\left(\frac{R^{2 m-1}}{R-r} T_{F}\left(R, r_{0}\right)\right)^{p^{\prime}} .
$$

Proof. By the property of the Wronskian (see [5], Proposition 4.9),

$$
W_{\alpha^{1} \ldots \alpha^{l}}\left(F_{1}, \ldots, F_{l}\right)=C W_{\alpha^{1} \ldots \alpha^{l}}\left(L_{1}(F), \ldots, L_{l}(F)\right),
$$

where $C$ is a constant depending only on $L_{1}, \ldots, L_{l}$. Hence, we only need to estimate

$$
I:=\int_{S(r)}\left|z^{\alpha^{1}+\cdots+\alpha^{l}} \frac{W_{\alpha^{1} \ldots \alpha^{l}}\left(L_{1}(F), \ldots, L_{l}(F)\right)}{L_{1}(F) \cdots L_{l}(F)}\right|^{t} \sigma_{m} .
$$

Let

$$
\chi=\frac{W_{\alpha^{1} \ldots \alpha^{l}}\left(L_{1}(F), \ldots, L_{l}(F)\right)}{L_{1}(F) \cdots L_{l}(F)}
$$

and set $\varphi_{j}:=L_{j}(F) / L_{1}(F), 1 \leq j \leq l$. Again, by the property of the Wronskian (see [5], Proposition 4.9), we can write $\chi$ as

$$
\chi=\frac{W_{\alpha^{1} \ldots \alpha^{l}}\left(\varphi_{1}, \varphi_{2}, \ldots, \varphi_{l}\right)}{\varphi_{1} \cdots \varphi_{l}}=\sum_{\sigma_{i_{1} \cdots i_{l}}} \operatorname{sgn}\left(\sigma_{i_{1} \cdots i_{l}}\right) \frac{D^{\alpha^{1}} \varphi_{i_{1}}}{\varphi_{i_{1}}} \cdots \frac{D^{\alpha^{l}} \varphi_{i_{l}}}{\varphi_{i_{l}}},
$$

where $\sigma_{i_{1} \cdots i_{l}}$ is the permutation from $(1,2, \ldots, l)$ to $\left(i_{1}, \ldots, i_{l}\right)$. Therefore, the above integrand can be estimated from above by a positive constant multiple of the sum of some functions of the type

$$
\psi_{i_{1} \ldots i_{l}}:=\left|z^{\alpha^{1}+\cdots+\alpha^{l}} \frac{D^{\alpha^{1}} \varphi_{i_{1}}}{\varphi_{i_{1}}} \cdots \frac{D^{\alpha^{l}} \varphi_{i_{l}}}{\varphi_{i_{l}}}\right|^{t} .
$$

Set $p_{j}=\frac{\left|\alpha^{j}\right|}{\left|\alpha^{1}\right|+\cdots+\left|\alpha^{l}\right|}$ for $1 \leq j \leq l$. By the Hölder inequality, we obtain

$$
\int_{S(r)} \psi_{i_{1} \ldots i_{l}} \sigma_{m} \leq \prod_{j=1}^{l}\left[\int_{S(r)}\left|z^{\alpha_{j}} \frac{D^{\alpha_{j}} \varphi_{i_{j}}}{\varphi_{i_{j}}}\right|^{t / p_{j}} \sigma_{m}\right]^{p_{j}} .
$$

Since $\left(t / p_{j}\right)\left|\alpha^{j}\right|=\left(\left|\alpha_{1}\right|+\cdots+\left|\alpha_{l}\right|\right) t<p^{\prime}<1$ for $1 \leq j \leq l$, we can apply Theorem 3.1 to get

$$
\int_{S(r)} \psi_{i_{1} \ldots i_{l}} \sigma_{m} \leq K \prod_{j=1}^{l}\left(\frac{R^{2 m-1}}{R-r} T_{\varphi_{i_{j}}}\left(R, r_{0}\right)\right)^{p^{\prime} p_{j}} .
$$

On the other hand, by (2.5) in [5],

$$
T_{\varphi_{j}}\left(r, r_{0}\right) \leq T_{F}\left(r, r_{0}\right)+K
$$

for every $i=1, \ldots, l$. Therefore, we conclude that

$$
I \leq K\left(\frac{R^{2 m-1}}{R-r} T_{F}\left(R, r_{0}\right)\right)^{p^{\prime}} .
$$

This completes the proof of the proposition. 


\section{Proof of the Main theorem}

In this section, we prove the main theorem (Theorem 1.1). Before doing that, we first prove some preparation lemmas.

Let $D_{1}, \ldots, D_{q}$ be hypersurfaces in $\mathbb{P}^{n}(\mathbb{C})$ of degree $d$, located in general position, and let $Q_{j}, 1 \leq j \leq q$, be homogeneous polynomials defining $D_{j}$. Let $N$ be a large integer (to be determined later), and let $V_{N}$ be the space of homogeneous polynomials of $n+1$ variables of degree $N$. Pick $n$ distinct polynomials $\gamma_{1}, \ldots, \gamma_{n} \in$ $\left\{Q_{1}, \ldots, Q_{q}\right\}$. Arrange the $n$-tuples $\mathbf{i}=\left(i_{1}, \ldots, i_{n}\right)$ of non-negative integers by lexicographic order. Define, for the $n$-tuples $\mathbf{i}=\left(i_{1}, \ldots, i_{n}\right)$ of non-negative integers with $\sigma(\mathbf{i}):=\sum_{j} i_{j} \leq N / d$, the spaces $W_{\mathbf{i}}:=W_{N, \mathbf{i}}$ by

$$
W_{N, \mathbf{i}}=\sum_{\mathbf{e} \geq \mathbf{i}} \gamma_{1}^{e_{1}} \cdots \gamma_{n}^{e_{n}} V_{N-d \sigma(\mathbf{e})} .
$$

Clearly, $W_{(0, \ldots, 0)}=V_{N}$ and $W_{\mathbf{i}} \supset W_{\left(\mathbf{i}^{\prime}\right)}$ if $\mathbf{i}^{\prime} \geq \mathbf{i}$, so that the $\left\{W_{\mathbf{i}}\right\}$ in fact define a filtration of $V_{N}$. We recall the following lemma due to 3 .

Lemma 4.1 (See [3], Proposition 3.3). For any non-negative integer $N$ and any $\left\{\gamma_{1}, \ldots, \gamma_{n}\right\} \subset\left\{Q_{1}, \ldots, Q_{q}\right\}$, the dimension of the vector space $\frac{V_{N}}{\left(\gamma_{1}, \ldots, \gamma_{n}\right) \cap V_{N}}$ is equal to the number of $n$-tuples $(\mathbf{i})=\left(i_{1}, \ldots, i_{n}\right) \in \mathbb{Z}_{\geq 0}^{n}$ such that $i_{1}+\cdots+i_{n} \leq N$ and $0 \leq i_{1}, \ldots, i_{n} \leq d-1$. In particular, for all $N \geq n(d-1)$, we have

$$
\operatorname{dim} \frac{V_{N}}{\left(\gamma_{1}, \ldots, \gamma_{n}\right) \cap V_{N}}=d^{n}
$$

Lemma 4.2 (See [10], Lemma 3.2). There is an isomorphism

$$
\frac{W_{\mathbf{i}}}{W_{\mathbf{i}^{\prime}}} \cong \frac{V_{N-d \sigma(\mathbf{i})}}{\left(\gamma_{1}, \ldots, \gamma_{n}\right) \cap V_{N-d \sigma(\mathbf{i})}},
$$

where $\mathbf{i}^{\prime}>\mathbf{i}$ are consecutive $n$-tuples with $W_{\mathbf{i}}^{\prime} \subset W_{\mathbf{i}}$.

Let $\triangle_{\mathbf{i}}=\operatorname{dim}\left(W_{\mathbf{i}} / W_{\mathbf{i}^{\prime}}\right)$, where $\mathbf{i}^{\prime}>\mathbf{i}$ are consecutive $n$-tuples with $W_{\mathbf{i}^{\prime}} \subset W_{\mathbf{i}}$. By Lemma 4.1, $\triangle_{\mathbf{i}}=d^{n}$ for every $\mathbf{i}$ such that $N-d \sigma(\mathbf{i}) \geq n(d-1)$. Moreover, Lemma 4.1 implies that $\triangle_{\mathbf{i}}$ is independent of the choice of $\gamma_{1}, \ldots, \gamma_{n}$. Hence, $\sum_{\mathbf{i}} i_{j} \triangle_{\mathbf{i}}$ is independent of the choice of $\gamma_{1}, \ldots, \gamma_{n}$ and $j$ for $j=1, \ldots, n$. Set, for $1 \leq j \leq n$,

$$
\triangle:=\sum_{\mathbf{i}} i_{j} \triangle_{\mathbf{i}}
$$

Lemma 4.3. With $N=2 d(n+1)(n d+n)\left(2^{n}-1\right)\left(I\left(\epsilon^{-1}\right)+1\right)+n d$ for any $\epsilon>0$, we have

$$
\frac{l N}{\triangle} \leq d(n+1)+\epsilon / 2
$$

where $l=\left(\begin{array}{c}N+n \\ n\end{array}\right)$. Moreover, $l$ satisfies the following estimate:

$$
l \leq 2^{n^{2}+4 n} e^{n} d^{2 n}\left(n I\left(\epsilon^{-1}\right)\right)^{n},
$$

where $I(x):=\min \{k \in \mathbb{N}: k>x\}$ for a positive real number $x$. 
Proof. First notice that

$$
l=\left(\begin{array}{c}
N+n \\
n
\end{array}\right)=\frac{(n+N)(n+N-1) \cdots(n+1) N !}{N ! n !} \leq \frac{(N+n)^{n}}{n !} .
$$

Now since $N$ is divisible by $d$, it follows from Lemma 4.1 that

$$
\begin{aligned}
\triangle=\sum_{\sigma(\mathbf{i}) \leq N / d} i_{j} \triangle_{\mathbf{i}} & \geq \sum_{\sigma(\mathbf{i}) \leq N / d-n} i_{j} \triangle_{\mathbf{i}}=d^{n} \sum_{\sigma(\mathbf{i}) \leq N / d-n} i_{j} \\
& =\frac{d^{n}}{n+1} \sum_{\sigma(\hat{\mathbf{i}})=N / d-n} \sum_{j=1}^{n+1} i_{j} \\
& =\frac{d^{n}}{n+1} \sum_{\sigma(\hat{\mathbf{i}})=N / d-n}(N / d-n) \\
& =\frac{d^{n}}{n+1}\left(\begin{array}{c}
N / d \\
n
\end{array}\right)(N / d-n) \\
& =\frac{N(N-d) \cdots(N-d n)}{d(n+1) !},
\end{aligned}
$$

where $\hat{\mathbf{i}}=\left(i_{1}, \ldots, i_{n+1}\right)$ and, in the above, we used the fact that the number of non-negative integer $m$-tuples with sum $\leq T$ for a positive integer $T$ is equal to the number of non-negative integer $(m+1)$-tuples with sum exactly $T$, which is $\left(\begin{array}{c}T+m \\ m\end{array}\right)$.

For every integer $j \leq n,(N-d j) \geq(N-d n)$; so

$$
\prod_{j=1}^{n} \frac{1}{N-d j} \leq\left(\frac{1}{N-d n}\right)^{n}
$$

and thus

$$
\frac{l N}{\triangle} \leq d(n+1)\left(\frac{N+n}{N-n d}\right)^{n}
$$

Using

$$
N=2 d(n+1)(n d+n)\left(2^{n}-1\right)\left(I\left(\epsilon^{-1}\right)+1\right)+n d,
$$

one finds that

$$
\begin{aligned}
\left(\frac{N+n}{N-n d}\right)^{n} & =\left(1+\frac{n+n d}{N-n d}\right)^{n} \\
& =1+\sum_{r=1}^{n}\left(\begin{array}{c}
n \\
r
\end{array}\right)\left(\frac{n d+n}{N-n d}\right)^{r} \\
& \leq 1+\left(2^{n}-1\right) \frac{n d+n}{N-n d} \\
& \leq 1+\frac{\epsilon}{2 d(n+1)} .
\end{aligned}
$$

Therefore

$$
\frac{l N}{\triangle} \leq d(n+1)+\epsilon / 2 .
$$


To estimate $l$, we use the following inequality:

$$
\left(\begin{array}{c}
x+y \\
y
\end{array}\right) \leq \frac{(x+y)^{x+y}}{x^{x} y^{y}}=\left(1+\frac{y}{x}\right)^{x}\left(1+\frac{x}{y}\right)^{y}=\left(e\left(1+\frac{x}{y}\right)\right)^{y}
$$

for positive integers $x, y$. Hence, with $N=2 d(n+1)(n d+n)\left(2^{n}-1\right)\left(I\left(\epsilon^{-1}\right)+1\right)+n d$, we have

$$
\begin{aligned}
l & =\left(\begin{array}{c}
N+n \\
n
\end{array}\right) \leq e^{n}\left(1+\frac{N}{n}\right)^{n} \\
& \leq e^{n}\left(1+2 d(n+1)(d+1)\left(2^{n}-1\right)\left(I\left(\epsilon^{-1}\right)+1\right)+d\right)^{n} \\
& \leq 2^{n^{2}+4 n} e^{n} d^{2 n}\left(n I\left(\epsilon^{-1}\right)\right)^{n} .
\end{aligned}
$$

We now prove the main theorem. Since the universal covering of $M$ is the unit ball in $\mathbb{C}^{m}$, by lifting $f$ to the covering, we may assume that $M=B(1) \subset \mathbb{C}^{m}$. So we let $f: B(1) \longrightarrow \mathbb{P}^{n}(\mathbb{C})$ be an algebraically non-degenerate map. The proof of the main theorem breaks into the following two cases: the case

$$
\lim _{r \longrightarrow 1} \sup \frac{T_{f}\left(r, r_{0}\right)}{\log 1 /(1-r)}<\infty
$$

and the case

$$
\lim _{r \longrightarrow 1} \sup \frac{T_{f}\left(r, r_{0}\right)}{\log 1 /(1-r)}=\infty .
$$

We first deal with the case when

$$
\lim _{r \longrightarrow 1} \sup \frac{T_{f}\left(r, r_{0}\right)}{\log 1 /(1-r)}<\infty .
$$

Let $D_{1}, \ldots, D_{q}$ be hypersurfaces in $\mathbb{P}^{n}(\mathbb{C})$ of degree $d_{1}, \ldots, d_{q}$, located in general position. Let $Q_{j}, 1 \leq j \leq q$, be the homogeneous polynomials defining $D_{j}$. Replacing $Q_{j}$ by $Q_{j}^{d / d_{j}}$ if necessary, where $d$ is the l.c.m. (the least common multiple) of the $d_{j}$ 's, we can assume that $Q_{1}, \ldots, Q_{q}$ have the same degree $d$. For $N \in \mathbb{N}$, let $V_{N}$ be the space of homogeneous polynomials of $n+1$ variables of degree $N$ and fix a (arbitrary) basis $\phi_{1}, \ldots, \phi_{l}$, where $l=\operatorname{dim} V_{N}$. Set $F=\left[\phi_{1}(f): \cdots: \phi_{l}(f)\right]$. Then $F: B(1) \rightarrow \mathbb{P}^{l-1}(\mathbb{C})$ is linearly non-degenerate. By Proposition 3.2 there exist $\alpha^{j}=\left(\alpha_{j 1}, \ldots, \alpha_{j l}\right)$ with $\alpha_{j i} \geq 0$ being integers, $\left|\alpha^{j}\right| \leq l-1$ for $1 \leq j \leq l$, and $\left|\alpha^{1}\right|+\cdots+\left|\alpha^{l}\right| \leq l(l-1) / 2$ such that the generalized Wronskian $W_{\alpha^{1} \cdots \alpha^{l}}(F) \not \equiv 0$.

Given $z \in B(1)$ there exists a numbering $\left\{i_{1}, \ldots, i_{q}\right\}$ of the indices $1, \ldots, q$ such that

$$
\left|Q_{i_{1}} \circ f(z)\right| \leq \cdots \leq\left|Q_{i_{q}} \circ f(z)\right| .
$$

Since $Q_{1}, \ldots, Q_{q}$ are in general position, the Hilbert Nullstellensatz implies that for any integer $k, 0 \leq k \leq n$, there is an integer $m_{k} \geq d$ such that

$$
x_{k}^{m_{k}}=\sum_{j=1}^{n+1} b_{j k}\left(x_{0}, \ldots, x_{n}\right) Q_{i_{j}}\left(x_{0}, \ldots, x_{n}\right),
$$

where $b_{j k}, 1 \leq j \leq n+1,0 \leq k \leq n$, are homogeneous forms with coefficients in $\mathbb{C}$ of degree $m_{k}-d$. So

$$
\left|f_{k}(z)\right|^{m_{k}} \leq c_{1}\|f(z)\|^{m_{k}-d} \max \left\{\left|Q_{i_{1}}(f)(z)\right|, \ldots,\left|Q_{i_{n+1}}(f)(z)\right|\right\},
$$


where $c_{1}$ is a positive constant depending only on the coefficients of $b_{j k}$, thus depending only on the coefficients of $Q_{j}$. Therefore,

$$
\|f(z)\|^{d} \leq c_{1} \max \left\{\left|Q_{i_{1}}(f)(z)\right|, \ldots,\left|Q_{i_{n+1}}(f)(z)\right|\right\} .
$$

By (4.5) and (4.6), we get

$$
\prod_{j=1}^{q} \frac{\|f(z)\|^{d}}{\left|Q_{j}(f)(z)\right|} \leq c_{1}^{q-n} \prod_{k=1}^{n} \frac{\|f(z)\|^{d}}{\left|Q_{i_{k}}(f)(z)\right|} .
$$

Take $\gamma_{1}=Q_{i_{1}}, \cdots, \gamma_{n}=Q_{i_{n}}$ and let $V_{N}=W_{\mathbf{0}} \supset \cdots \supset W_{\mathbf{i}} \supset W_{\mathbf{i}^{\prime}} \supset \cdots$ be the filtration of $V_{N}$, associated to $\left\{\gamma_{1}, \ldots, \gamma_{n}\right\}$ as discussed earlier. We now choose a basis $\psi_{1}, \ldots, \psi_{l}$ for $V_{N}$ in the following way: We start with the last non-zero $W_{\mathbf{i}}$ and pick a basis of it. Then, we continue inductively as follows: suppose $\mathbf{i}^{\prime}>\mathbf{i}$ are consecutive $n$-tuples such that $d \sigma(\mathbf{i}), d \sigma\left(\mathbf{i}^{\prime}\right) \leq N$ and assume that we have chosen a basis of $W_{\left(\mathbf{i}^{\prime}\right)}$. It follows directly from the definition that we may pick representatives in $W_{\mathbf{i}}$ for the quotient space $W_{\mathbf{i}} / W_{\mathbf{i}^{\prime}}$, of the form $\gamma_{1}^{i_{1}} \cdots \gamma_{n}^{i_{n}} \eta$, where $\eta \in V_{N-d \sigma(\mathbf{i})}$. We extend the previously constructed basis in $W_{\mathbf{i}^{\prime}}$ by adding these representatives. In particular we have obtained a basis for $W_{\mathbf{i}}$ and our induction procedure may go on unless $W_{\mathbf{i}}=V_{N}$. Note that if we let $\psi$ be an element of the basis constructed with respect to $W_{\mathbf{i}} / W_{\mathbf{i}^{\prime}}$, then we may write $\psi=\gamma_{1}^{i_{1}} \cdots \gamma_{n}^{i_{n}} \eta$, where $\eta \in V_{N-d \sigma(\mathbf{i})}$. Thus we have a bound

$$
|\psi(f)(z)| \leq c_{2}\left|\gamma_{1}(f)(z)\right|^{i_{1}} \cdots\left|\gamma_{n}(f)(z)\right|^{i_{n}}\|f(z)\|^{N-d \sigma(i)},
$$

where $c_{2}$ is a positive constant which depends only on $f$ and $Q_{1}, \ldots, Q_{q}$. Observe that there are precisely $\triangle_{\mathbf{i}}$ such functions $\psi$ in our basis. Write $\psi_{1}, \ldots, \psi_{l}$ as linear forms $L_{1}, \ldots, L_{l}$ in $\phi_{1}, \ldots, \phi_{l}$ so that $\psi_{t}(f)=L_{t}(F)$, where $F=\left[\phi_{1}(f): \cdots: \phi_{l}(f)\right]$. Then (4.8) implies that

$$
\prod_{t=1}^{l}\left|L_{t}(F(z))\right| \leq K\left(\prod_{\mathbf{i}=\left(i_{1}, \ldots, i_{n}\right)}\left|\gamma_{1}^{i_{1}}(f(z)) \cdots \gamma_{n}^{i_{n}}(f(z))\right|^{\triangle_{\mathbf{i}}}\right)\|f(z)\|^{l N-d \sum_{\mathbf{i}} \sigma(\mathbf{i}) \triangle_{\mathbf{i}}},
$$

where, as we noted earlier, $K$ is a constant depending only on $f$ and $D_{1}, \ldots, D_{q}$ which may be different each time. So

$$
\frac{\|f(z)\|^{d \sum_{\mathbf{i}} \sigma\left(\mathbf{i} \triangle_{\mathbf{i}}\right.}}{\prod_{\mathbf{i}}\left|\gamma_{1}^{i_{1} \triangle_{\mathbf{i}}}(f(z))\right| \cdots\left|\gamma_{n}^{i_{n} \triangle_{\mathbf{i}}}(f(z))\right|} \leq K \frac{\|f(z)\|^{l N}}{\prod_{t=1}^{l}\left|L_{t}(F(z))\right|}
$$

thus, using (4.1),

$$
\frac{\|f(z)\|^{d n \triangle}}{\left|\gamma_{1}^{\triangle}(f(z))\right| \cdots\left|\gamma_{n}^{\triangle}(f(z))\right|} \leq K \frac{\|f(z)\|^{l N}}{\prod_{t=1}^{l}\left|L_{t}(F(z))\right|} .
$$

With $\gamma_{1}=Q_{i_{1}}, \cdots, \gamma_{n}=Q_{i_{n}}$, this gives

$$
\frac{\|f(z)\|^{d n \triangle}}{\left|Q_{i_{1}}^{\triangle}(f(z)) \cdots Q_{i_{n}}^{\triangle}(f(z))\right|} \leq K \frac{\|f(z)\|^{l N}}{\prod_{t=1}^{l}\left|L_{t}(F(z))\right|} .
$$

On the other hand, from (4.7), we get

$$
\frac{\|f(z)\|^{d q \Delta}}{\left|Q_{1}^{\triangle}(f(z)) \cdots Q_{q}^{\triangle}(f(z))\right|} \leq K \frac{\|f(z)\|^{d n \triangle}}{\left|Q_{i_{k}}^{\triangle}(f)(z) \cdots Q_{i_{n}}^{\triangle}(f)(z)\right|} .
$$


Combining (4.9) and (4.10), we derive that

$$
\frac{\|f(z)\|^{d q \triangle}}{\left|Q_{1}^{\triangle}(f(z)) \cdots Q_{q}^{\triangle}(f(z))\right|} \leq K \frac{\|f(z)\|^{l N}}{\prod_{t=1}^{l}\left|L_{t}(F(z))\right|} .
$$

Hence,

$$
\frac{\|f(z)\|^{d q \triangle-l N}\left|W_{\alpha^{1} \ldots \alpha^{l}}(F)(z)\right|}{\left|Q_{1}^{\triangle}(f(z)) \cdots Q_{q}^{\triangle}(f(z))\right|} \leq K \frac{\left|W_{\alpha^{1} \ldots \alpha^{l}}(F)(z)\right|}{\left|L_{1}(F(z)) \cdots L_{l}(F(z))\right|} .
$$

Note that although $L_{1}, \ldots, L_{l}$ depend on $z$, there are only finitely many such choices since there are only finitely many choices of $\left\{\gamma_{1}, \ldots, \gamma_{n}\right\} \subset\left\{Q_{1}, \ldots, Q_{q}\right\}$.

We continue with the proof of the main theorem by absurdity. We assume that

$$
\rho \Omega_{f}+\frac{\sqrt{-1}}{2 \pi} \partial \bar{\partial} \log h^{2} \geq \operatorname{Ric} \omega
$$

and

$$
\sum_{j=1}^{q} \delta_{l-1}^{f}\left(D_{j}\right)>(n+1)+\epsilon+\frac{\rho l(l-1)}{d} .
$$

Then, from the discussion earlier, there exist constants $\eta_{j} \geq 0$ and continuous plurisubharmonic functions $\tilde{u}_{j}(\not \equiv-\infty)$ such that $e^{\tilde{u}_{j}}\left|\varphi_{j}\right| \leq\|f\|^{d \eta_{j}}$ for $j=1, \ldots, q$, and

$$
q-\sum_{j=1}^{q} \eta_{j}>n+1+\epsilon+\frac{\rho l(l-1)}{d},
$$

where $\varphi_{j}$ is a non-zero holomorphic function with $\nu_{\varphi_{j}}^{0}=\min \left(\nu^{f}\left(D_{j}\right), l-1\right)$. Let $u_{j}=\tilde{u}_{j}+\log \left|\varphi_{j}\right|$. Then, $u_{j}(\not \equiv-\infty), 1 \leq j \leq q$, are continuous plurisubharmonic functions,

$$
e^{u_{j}} \leq\|f\|^{d \eta_{j}}
$$

and $u_{j}-\log \left|\varphi_{j}\right|$ is plurisubharmonic, where $\varphi_{j}$ is a non-zero holomorphic function with $\nu_{\varphi_{j}}^{0}=\min \left(\nu^{f}\left(D_{j}\right), l-1\right)$. Let

$$
v:=\log \left|z^{\alpha^{1}+\cdots+\alpha^{l}} \frac{W_{\alpha^{1} \ldots \alpha^{l}}(F)}{Q_{1}^{\triangle}(f) \cdots Q_{q}^{\triangle}(f)}\right|+\sum_{j=1}^{q} \triangle u_{j},
$$

where $\triangle$ is the integer defined in (4.1). We now show that $v$ is plurisubharmonic on $M=B(1)$. To do so, we need the following lemma.

Proposition 4.4. In the above situation, set

$$
\psi=\frac{W_{\alpha^{1} \cdots \alpha^{l}}(F)}{Q_{1}^{\triangle}(f) \cdots Q_{q}^{\triangle}(f)} .
$$

Then

$$
\nu_{\psi}^{\infty} \leq \sum_{j=1}^{q} \triangle \min \left\{\nu_{Q_{j}(f)}^{0}, l-1\right\}
$$

outside an analytic set of codimension at least two. 
Proof. Let $I_{F}$ be the indeterminacy set of $F$, and take $a \in B(1) \backslash I_{F}$. We first show the following claim: If $h$ is a holomorphic function around $a$, assume that $D^{\alpha} h \neq \equiv$ around $a$. Then $\nu_{D^{\alpha} h}^{0}(a)=\max \left\{0, \nu_{h}^{0}(a)-|\alpha|\right\}$. To see this, take a system of holomorphic local coordinates $z=\left(z_{1}, \ldots, z_{m}\right)$ in a neighborhood of $a$ such that $z(a)=0$ and $h$ can be written as $h=z_{1}^{\nu_{h}^{0}(a)} \tilde{h}$, and $\tilde{h}$ has no zero in a neighborhood of $a$. From this representation of $h$, we can easily conclude the claim.

Now for each $a \in B(1) \backslash I_{F}$, without loss of generality, we may assume that $Q_{j}(f)$ vanishes at $a$ for $1 \leq j \leq q_{1}$ and that $Q_{j}(f)$ does not vanish at $a$ for $j>q_{1}$. By the assumption that the $Q_{j}$ 's are in general position, we know that $q_{1} \leq n$.

For $\left\{Q_{1}, \ldots, Q_{n}\right\} \subset\left\{Q_{1}, \ldots, Q_{q}\right\}$, consider the filtration $V_{N}=W_{\mathbf{0}} \supset \cdots \supset W_{\mathbf{i}} \supset$ $W_{\mathbf{i}^{\prime}} \supset \cdots$, associated to $\left\{Q_{1}, \ldots, Q_{n}\right\}$ as discussed earlier, and take a basis $\psi_{1}, \ldots, \psi_{l}$ of $V_{N}$ according to this filtration. Then, there are linearly independent linear forms $L_{1}, \ldots, L_{l}$ such that $\psi_{t}(f)=L_{t}(F), 1 \leq t \leq l$. Denote by $W:=W_{\alpha^{1} \ldots \alpha^{l}}(F)$, the generalized Wronskian of $F$. From the basic properties of the generalized Wronskian (see [5] Proposition 4.9),

$$
W=W_{\alpha^{1} \ldots \alpha^{l}}(F)=C W_{\alpha^{1} \ldots \alpha^{l}}\left(L_{1}(F), \ldots, L_{l}(F)\right)=C W_{\alpha^{1} \ldots \alpha^{l}}\left(\psi_{1}(f), \ldots, \psi_{l}(f)\right),
$$

where $C$ is some constant. Let $\psi$ be an element of the basis $\left\{\psi_{1}, \ldots, \psi_{l}\right\}$. As we discussed earlier, we may write $\psi=Q_{1}^{i_{1}} \cdots Q_{n}^{i_{n}} \eta$ with $\eta \in V_{N-d \sigma(i)}$. Therefore

$$
\psi(f)=\left(Q_{1}(f)\right)^{i_{1}} \cdots\left(Q_{n}(f)\right)^{i_{n}} \eta(f),
$$

and note that there are $\triangle_{\mathbf{i}}$ such that $\psi$ is our basis. Assume that $\nu_{Q_{j}(f)}^{0}(a) \geq l-1$ for $1 \leq j \leq q_{0}$ and $\nu_{Q_{j}(f)}^{0}(a)<l-1$ for $q_{0}<j \leq q_{1}$. Since, from above, $W=$ $C \operatorname{det}\left(D^{\alpha^{i}}\left(\psi_{j}(f)\right)\right)_{1 \leq i, j \leq l}$, by the claim (note that there are $\triangle_{\mathbf{i}}$ such that $\psi$ is our basis), and noticing that $\left|\alpha^{j}\right| \leq l-1$ for $1 \leq j \leq l$,

$$
\begin{aligned}
\nu_{W}^{0}(a) & \geq \sum_{\mathbf{i}}\left(\sum_{j=1}^{q_{0}} i_{j}\left(\nu_{Q_{j}}^{0}(a)-(l-1)\right)\right) \triangle_{\mathbf{i}} \\
& =\sum_{j=1}^{q_{0}}\left(\sum_{\mathbf{i}} i_{j} \triangle_{\mathbf{i}}\right)\left(\nu_{Q_{j}}^{0}(a)-(l-1)\right)=\triangle \sum_{j=1}^{q_{0}}\left(\nu_{Q_{j}}^{0}(a)-(l-1)\right) .
\end{aligned}
$$

On the other hand,

$$
\sum_{j=1}^{q} \nu_{Q_{j}(f)}^{0}(a)=\sum_{j=1}^{n} \nu_{Q_{j}(f)}^{0}(a)=\sum_{j=1}^{q_{0}} \nu_{Q_{j}(f)}^{0}(a)+\sum_{j=q_{0}}^{q_{1}} \nu_{Q_{j}(f)}^{0}(a) .
$$

Hence, $\nu_{\psi}^{\infty}(a) \leq \sum_{j=0}^{q} \triangle \min \left\{\nu_{Q_{j}(f)}(a), l-1\right\}$.

From the above proposition, by the definition of $v$ (see (4.16) ), and using the fact that $u_{j}-\log \left|\varphi_{j}\right|$ is plurisubharmonic and $\nu_{\varphi_{j}}^{0}=\min \left(\nu^{f}\left(D_{j}\right), l-1\right)$, we see that $v$ is plurisubharmonic on $M=B(1)$.

We now continue our proof. By the growth condition of $f$ (see (4.12) $)$, there exists a continuous plurisubharmonic function $w \not \equiv-\infty$ on $B(1)$ such that

$$
e^{w} d V \leq\|f\|^{2 \rho} v_{m}
$$

Set

$$
t=\frac{2 \rho}{q d \triangle-l N-\triangle d\left(\eta_{1}+\cdots+\eta_{q}\right)}
$$


and

$$
\chi:=z^{\alpha^{1}+\cdots+\alpha^{l}} \frac{W_{\alpha^{1} \ldots \alpha^{l}}(F)}{Q_{1}^{\triangle}(f) \cdots Q_{q}^{\triangle}(f)} .
$$

Define

$$
u:=w+t v .
$$

Then $u$ is plurisubharmonic and so subharmonic on the Kähler manifold $M$.

By the result of S.-T. Yau ([13]) and L. Karp ([]), we have necessarily

$$
\int_{B(1)} e^{u} d V=\infty
$$

because $B(1)$ has infinite volume with respect to the given complete Kähler metric (cf. [8], Theorem B). Now, from (4.15), (4.17) and (4.18),

$$
\begin{aligned}
e^{u} d V & =e^{w+t v} d V \leq e^{t v}\|f\|^{2 \rho} v_{m} \\
& =|\chi|^{t}\left(\prod_{j=1}^{q} e^{t \triangle u_{j}}\right)\|f\|^{2 \rho} v_{m} \leq|\chi|^{t}\left(\prod_{j=1}^{q}\|f\|^{t \triangle d \eta_{j}}\right)\|f\|^{2 \rho} v_{m} \\
& =|\chi|^{t}\|f\|^{2 \rho+t d \Delta \sum_{j=1}^{q} \eta_{j}} v_{m}=|\chi|^{t}\|f\|^{t(d q \triangle-l N)} v_{m} .
\end{aligned}
$$

The contradiction will appear if we can show that

$$
\int_{B(1)} e^{u} d V<\infty
$$

From Lemma 4.3 $\frac{l N}{\triangle} \leq d(n+1)+\epsilon$. Thus $q d-\frac{l N}{\triangle} \geq d(q-(n+1+\epsilon))$. So, using (4.14),

$$
d q \triangle-l N-\triangle \sum_{j=1}^{q} d \eta_{j} \geq d \triangle(q-(n+1+\epsilon))-\triangle \sum_{j=1}^{q} d \eta_{j}>\triangle \rho l(l-1) .
$$

This implies that $t l(l-1) / 2<1$. Since $\left|\alpha^{1}\right|+\cdots+\left|\alpha^{l}\right| \leq l(l-1) / 2$, we can choose $p^{\prime}$ such that $t\left(\left|\alpha^{1}\right|+\cdots+\left|\alpha^{l}\right|\right) \leq t l(l-1) / 2<p^{\prime}<1$. By the help of the identity (cf. [12], p.226)

$$
v_{m}=\left(d d^{c}|z|^{2}\right)^{m}=2 m|z|^{2 m-1} \sigma_{m} \wedge d|z|,
$$

we have

$$
\begin{aligned}
\int_{B(1)} e^{u} d V & \leq \int_{B(1)}|\chi|^{t}\|f\|^{t(d q \triangle-l N)} v_{m} \\
& \leq 2 m \int_{0}^{1} r^{2 m-1}\left(\int_{S(r)}|\chi|^{t}\|f\|^{t(d q \triangle-l N)} \sigma_{m}\right) d r \\
& =2 m \int_{0}^{1} r^{2 m-1}\left(\int_{S(r)}\left|z^{\alpha^{1}+\cdots+\alpha^{l}} \frac{W_{\alpha^{1} \ldots \alpha^{l}}(F)\|f\|^{(d q \triangle-l N)}}{Q_{1}^{\triangle}(f) \cdots Q_{q}^{\triangle}(f)} \sigma_{m}\right|^{t}\right) d r .
\end{aligned}
$$

On the other hand, by (4.11),

$$
\frac{\left|W_{\alpha^{1} \ldots \alpha^{l}}(F)\right|\|f\|^{(d q \triangle-l N)}}{\left|Q_{1}^{\triangle}(f) \cdots Q_{q}^{\triangle}(f)\right|} \leq K \sum_{L_{1}, \ldots, L_{l}}\left(\frac{\left|W_{\alpha^{1} \ldots \alpha^{l}}(F)\right|}{\left|L_{1}(F) \cdots L_{l}(F)\right|}\right),
$$

where the summation is taken for all the possible linear forms choices of the linear forms $L_{1}, \ldots, L_{l}$. Note that the set of linear forms $\left\{L_{1}, \ldots, L_{l}\right\}$ comes from the 
filtration of $V_{N}$ associated to the $\left\{\gamma_{1}, \ldots, \gamma_{n}\right\} \subset\left\{Q_{1}, \ldots, Q_{q}\right\}$. Hence the number of choices of the sets $\left\{L_{1}, \ldots, L_{l}\right\}$ is the same as the number of the choices of the sets $\left\{\gamma_{1}, \ldots, \gamma_{n}\right\}$, which is finite. Hence the summation in (4.20) is a finite sum whose number of terms depends only on $f$ and $Q_{1}, \ldots, Q_{q}$. By Proposition 3.3 , for each $L_{1}, \ldots, L_{l}$,

$$
\int_{S(r)}\left|z^{\alpha^{1}+\cdots+\alpha^{l}} \frac{W_{\alpha^{1} \ldots \alpha^{l}}(F)}{L_{1}(F) \cdots L_{l}(F)}\right|^{t} \sigma_{m} \leq K\left(\frac{R^{2 m-1}}{R-r} T_{F}\left(R, r_{0}\right)\right)^{p^{\prime}} .
$$

Combining (4.20) and (4.21) thus gives

$$
\int_{S(r)}\left|z^{\alpha^{1}+\cdots+\alpha^{l}} \frac{W_{\alpha^{1} \ldots \alpha^{l}}(F)}{Q_{1}^{\triangle}(f) \cdots Q_{q}^{\triangle}(f)}\right|^{t}\|f\|^{t(d q \triangle-l N)} \sigma_{m} \leq K\left(\frac{R^{2 m-1}}{R-r} T_{F}\left(R, r_{0}\right)\right)^{p^{\prime}},
$$

for $r_{0}<r<R<1$, where, as we noted, we use the letter $K$ to denote a constant depending only on $f$ and $D_{1}, \ldots, D_{q}$ even when it should be replaced by a new constant. According to Lemma 2.4 in [7], if we choose $R:=r+(1-r) / e T_{F}\left(r, r_{0}\right)$, then

$$
T_{F}\left(R, r_{0}\right) \leq 2 T_{F}\left(r, r_{0}\right) \leq 2 d T_{f}\left(r, r_{0}\right)
$$

outside a set $E$ with $\int_{E} 1 /(1-r) d r<\infty$. If

$$
\limsup _{r \rightarrow 1} \frac{T_{f}\left(r, r_{0}\right)}{\log 1 /(1-r)}<\infty
$$

then (4.22) becomes

$$
\int_{S(r)}\left|z^{\alpha^{1}+\cdots+\alpha^{l}} \frac{W_{\alpha^{1} \ldots \alpha^{l}}(F)}{Q_{1}^{\triangle}(f) \cdots Q_{q}^{\triangle}(f)}\right|^{t}\|f\|^{t(d q \triangle-l N)} \sigma_{m} \leq \frac{K}{(1-r)^{p^{\prime}}}\left(\log \frac{1}{1-r}\right)^{p^{\prime}}
$$

for all $r \in[0,1)$ outside a set $E$ with $\int_{E} 1 /(1-r) d r<\infty$. Varying a constant $K$ slightly, we may assume that the above inequality holds for all $r \in[0,1)$ because of Proposition 5.5 in 5 . Therefore, by (4.19) and (4.23), we have

$$
\int_{B(1)} e^{u} d V \leq K \int_{0}^{1} \frac{r^{2 m-1}}{(1-r)^{p^{\prime}}}\left(\log \frac{1}{1-r}\right)^{p^{\prime}} d r<\infty
$$

since $p^{\prime}<1$. This contradicts the result of S.-T. Yau ([13]) and L. Karp ([8]) mentioned earlier. This completes the proof for the first case.

We now deal with the case where

$$
\limsup _{r \longrightarrow 1} \frac{T_{f}\left(r, r_{0}\right)}{\log 1 /(1-r)}=\infty .
$$

This case is similar to the standard Nevanlinna theory. We use the logarithmic derivative lemma and the previous discussions to prove the following refinement of the second main theorem (see [10]).

Theorem 4.5. Let $f: B\left(R_{0}\right) \longrightarrow \mathbb{P}^{n}(\mathbb{C}), 0<R_{0} \leq \infty$, be a meromorphic map which is algebraically non-degenerate and $D_{1}, \ldots, D_{q}$ be hypersurfaces of degree $d_{j}, 1 \leq j \leq q$, in $\mathbb{P}^{n}(\mathbb{C})$ located in general position. Then, for every $\epsilon>0$,

$$
(q-(n+1+\epsilon)) T_{f}\left(r, r_{0}\right) \leq \sum_{j=1}^{q} d_{j}^{-1} N_{f}^{[l-1]}\left(r, D_{j}\right)+S(r)
$$


where $l \leq 2^{n^{2}+4 n} e^{n} d^{2 n}\left(n I\left(\epsilon^{-1}\right)\right)^{n}, d=$ l.c.m. $\left\{d_{1}, \ldots, d_{q}\right\}$, and $S(r)$ is evaluated as follows:

(1) In the case $R_{0}<\infty$,

$$
S(r) \leq K\left(\log ^{+} \frac{1}{R_{0}-r}+\log ^{+} T_{f}\left(r, r_{0}\right)\right)
$$

for every $r \in\left[0, R_{0}\right)$ excluding a set $E$ with $\int_{E} \frac{1}{R_{0}-t} d t<\infty$.

(2) In the case $R_{0}=\infty$,

$$
S(r) \leq K\left(\log ^{+} T_{f}\left(r, r_{0}\right)+\log r\right)
$$

for every $r \in\left[0, R_{0}\right)$ excluding a set $E^{\prime}$ with $\int_{E^{\prime}} d t<\infty$.

Proof. Without loss of generality, we assume that $d_{1}=\cdots=d_{q}=d$. Similar to the proof of (4.22), by using (4.20) and Proposition 3.3, we have

$$
\int_{S(r)}\left|z^{\alpha^{1}+\cdots+\alpha^{l}} \frac{W_{\alpha^{1} \ldots \alpha^{l}}(F)}{Q_{1}^{\triangle}(f) \cdots Q_{q}^{\triangle}(f)}\right|^{t}\|f\|^{t(d q \triangle-l N)} \sigma_{m} \leq K\left(\frac{R^{2 m-1}}{R-r} T_{F}\left(R, r_{0}\right)\right)^{p^{\prime}}
$$

for $r_{0}<r<R<R_{0}$. Hence, by virtue of the concavity of the logarithm, the above inequality implies that

$$
\begin{aligned}
& t \int_{S(r)} \log \left|z^{\alpha^{1}+\cdots+\alpha^{l}}\right| \sigma_{m}+t \int_{S(r)} \log \left|\frac{W_{\alpha^{1} \ldots \alpha^{l}}(F)}{Q_{1}^{\triangle}(f) \cdots Q_{q}^{\triangle}(f)}\right| \sigma_{m} \\
& +t(d q \triangle-N l) \int_{S(r)} \log \|f\| \sigma_{m} \\
& \leq K\left(\log ^{+} \frac{R}{R-r}+\log ^{+} T_{F}\left(R, r_{0}\right)\right)+O(1),
\end{aligned}
$$

for $r_{0}<R<R_{0}$. But, by the Jensen formula (see [5], p.236),

$$
\int_{S(r)} \log \left|\frac{W_{\alpha^{1} \ldots \alpha^{l}}(F)}{Q_{1}^{\triangle}(f) \cdots Q_{q}^{\triangle}(f)}\right| \sigma_{m}=N_{\nu_{W_{\alpha^{1} \ldots \alpha^{l}}(F)}^{0}}\left(r_{0}, r\right)-\triangle \sum_{j=1}^{q} N_{f}\left(r, D_{j}\right)+O(1)
$$

By Proposition 4.4, $\triangle \sum_{j=1}^{q} N_{f}\left(r, D_{j}\right)-N_{\nu_{W_{\alpha^{1} \ldots \alpha^{l}}^{0}(F)}}\left(r_{0}, r\right) \leq \triangle \sum_{j=1}^{q} N_{f}^{[l-1]}\left(r, D_{j}\right)$ and therefore (4.25) becomes

$$
(d q \triangle-N l) T_{f}(r) \leq \sum_{j=1}^{q} \triangle N_{f}^{[l-1]}\left(r, D_{j}\right)+K\left(\log ^{+} \frac{R}{R-r}+\log ^{+} T_{F}\left(R, r_{0}\right)\right)+O(1)
$$

By Lemma 4.3, with $N=2 d(n+1)(n d+n)\left(2^{n}-1\right)\left(I\left(\epsilon^{-1}\right)+1\right)+n d$ for any $\epsilon>0$, we have

$$
\frac{l N}{\triangle} \leq d(n+1)+\epsilon
$$


and, moreover, $l$ satisfies $l \leq 2^{n^{2}+4 n} e^{n} d^{2 n}\left(n I\left(\epsilon^{-1}\right)\right)^{n}$. Hence,

$$
\begin{aligned}
(q-(n+1+\epsilon)) T_{f}(r) & \leq \sum_{j=1}^{q} d^{-1} N_{f}^{[l-1]}\left(r, D_{j}\right)+K\left(\log ^{+} \frac{R}{R-r}+\log ^{+} T_{F}\left(R, r_{0}\right)\right) \\
& \leq \sum_{j=1}^{q} d^{-1} N_{f}^{[l-1]}\left(r, D_{j}\right)+K\left(\log ^{+} \frac{R}{R-r}+\log ^{+} T_{f}\left(R, r_{0}\right)\right) .
\end{aligned}
$$

Since $T_{f}\left(r, r_{0}\right)$ is continuous, increasing and we may assume $T_{f}\left(r, r_{0}\right) \geq 1$, we can apply Lemma 2.4 in [7] to show that

$$
T_{f}\left(r+\frac{R_{0}-r}{e T_{f}\left(r, r_{0}\right)}, r_{0}\right) \leq 2 T_{f}\left(r, r_{0}\right)
$$

outside a set $E$ of $r$ such that $\int_{E} 1 /\left(R_{0}-r\right) d r<\infty$ in the case $R_{0}<\infty$ and

$$
T_{f}\left(r+\frac{1}{T_{f}\left(r, r_{0}\right)}, r_{0}\right) \leq 2 T_{f}\left(r, r_{0}\right)
$$

outside a set $E^{\prime}$ of $r$ such that $\int_{E^{\prime}} d r<\infty$ in the case $R_{0}=\infty$. Substituting $R=r+\frac{R_{0}-r}{e T_{f}\left(r, r_{0}\right)}$ if $R_{0}<\infty$ and $R=r+1 / T_{f}\left(r, r_{0}\right)$ if $R_{0}=\infty$ in (4.26) proves the theorem.

Corollary 4.6. In the same situation as in Theorem 4.5, if

$$
\text { (i) } \limsup _{r \rightarrow R_{0}} \frac{T_{f}\left(r, r_{0}\right)}{\log \left(1 / R_{0}-r\right)}=\infty
$$

or

$$
\text { (ii) } R_{0}=\infty \text {, }
$$

then

$$
\sum_{j} \delta_{l-1}^{f}\left(D_{j}\right) \leq \sum_{j} \delta_{l-1}^{f, \star}\left(D_{j}\right) \leq n+1+\epsilon,
$$

where $\delta^{f, \star}$ is the classical Nevanlinna's defect defined by

$$
\delta_{l-1}^{f, \star}\left(D_{j}\right)=\limsup _{r \rightarrow R_{0}}\left(1-\frac{N_{f}^{[l-1]}\left(r, D_{j}\right)}{d T_{f}\left(r, r_{0}\right)}\right) .
$$

Corollary 4.6 gives the proof of the second case. The proof of the main theorem (Theorem 1.1) is thus complete.

\section{Value Distribution of the Gauss map of a COMPlete Regular SUBMANIFOLD OF $\mathbb{C}^{m}$}

Let $f=\left(f_{1}, \ldots, f_{m}\right): M \rightarrow \mathbb{C}^{m}$ be a regular submanifold of $\mathbb{C}^{m}$; namely, let $M$ be a connected complex manifold and $f$ be a holomorphic map of $M$ into $\mathbb{C}^{m}$ such that rank $d_{p} f=\operatorname{dim} M$ for every point $p \in M$.

To each point $p \in M$, we assign the tangent space $T_{p}(M)$ of $M$ at $p$ which may be regarded as an $n$-dimensional linear subspace of $T_{f(p)} \mathbb{C}^{m}$. On the other hand, each $T_{p}\left(\mathbb{C}^{m}\right)$ is identified with $T_{0}\left(\mathbb{C}^{m}\right)=\mathbb{C}^{m}$ by a parallel translation. Therefore, to each $T_{p}(M)$ corresponds a point $G(p)$ in the complex Grassmannian manifold $G(n, m)$ of all $n$-dimensional linear subspaces of $\mathbb{C}^{m}$, where $n=\operatorname{dim} M$. 
Definition 5.1. We call the map $G: M \rightarrow G(n, m)$ the Gauss map of $f: M \rightarrow \mathbb{C}^{m}$.

The space $G(n, m)$ is canonically embedded in $\mathbb{P}^{N}(\mathbb{C})=\mathbb{P}\left(\bigwedge^{n} \mathbb{C}^{m}\right)$, where $N=\left(\begin{array}{l}m \\ n\end{array}\right)$ -1 . The Gauss map $G$ may be identified with a holomorphic map of $M$ into $\mathbb{P}^{N}(\mathbb{C})$ given as follows:

Taking holomorphic local coordinates $\left(z_{1}, \ldots, z_{n}\right)$ defined on an open set $U$, we consider the map

$$
\bigwedge:=D_{1} f \wedge \cdots \wedge D_{n} f: U \rightarrow \bigwedge^{n} \mathbb{C}^{m}-\{0\}
$$

where $D_{i} f=\left(\left(\partial / \partial z_{i}\right) f_{1}, \cdots,\left(\partial / \partial z_{i}\right) f_{N+1}\right)$. Then, locally we have

$$
G=\pi \circ \bigwedge
$$

where $\pi: \mathbb{C}^{N+1}-\{0\} \longrightarrow \mathbb{P}^{N}(\mathbb{C})$ is the canonical projection map.

A regular submanifold $M$ of $\mathbb{C}^{m}$ is considered a Kähler manifold with the metric $\omega$ induced from the standard flat metric on $\mathbb{C}^{m}$. By $d V$ we denote the volume form on $M$. We can see that, for arbitrarily holomorphic coordinates $z_{1}, \ldots, z_{n}$,

$$
d V=|\wedge|^{2}\left(\frac{\sqrt{-1}}{2}\right)^{n} d z_{1} \wedge d \overline{z_{1}} \wedge \cdots \wedge d z_{n} \wedge d \overline{z_{n}},
$$

where

$$
|\bigwedge|^{2}=\sum_{1 \leq i_{1}<\cdots<i_{n} \leq m}\left|\frac{\partial\left(f_{i_{1}}, \ldots, f_{i_{n}}\right)}{\partial\left(z_{1}, \ldots, z_{n}\right)}\right|^{2} .
$$

Therefore, for a regular submanifold $f: M \rightarrow \mathbb{C}^{m}$, the Gauss map $G: M \rightarrow \mathbb{P}^{N}(\mathbb{C})$ satisfies the following growth condition:

$$
\Omega_{G}+d d^{c} \log h^{2}=d d^{c} \log |\bigwedge|^{2}=\operatorname{Ric}(\omega),
$$

where $h=1$.

As a direct consequence of Theorem 1.1 we have

Theorem 5.2. Let $f: M \rightarrow \mathbb{C}^{m}$ be a complete regular submanifold such that the universal covering of $M$ is biholomorphic to $B\left(R_{0}\right) \quad\left(0<R_{0} \leq+\infty\right)$. If the Gauss map $G: M \rightarrow \mathbb{P}^{N}(\mathbb{C})$ is algebraically non-degenerate, then for all hypersurfaces $D_{1}, \ldots, D_{q}$ of degree $d_{j}, j=1, \ldots, q$ in general position, by letting $d=$ l.c.m. $\left\{d_{1}, \ldots, d_{q}\right\}$ (the least common multiple of $\left\{d_{1}, \ldots, d_{q}\right\}$ ), we have, for every $\epsilon>0$,

$$
\sum_{j=1}^{q} \delta_{l-1}^{G}\left(D_{j}\right) \leq N+1+\epsilon+\frac{l(l-1)}{d},
$$

where $l \leq 2^{N^{2}+4 N} e^{N} d^{2 N}\left(N I\left(\epsilon^{-1}\right)\right)^{N}, n=\operatorname{dim} M$, and $N=\left(\begin{array}{c}m \\ n\end{array}\right)-1$.

\section{REFERENCES}

[1] T. T. H. An and H. T. Phuong, An explicit estimate on multiplicity truncation in the second main theorem for holomorphic curves encountering hypersurfaces in general position in projective space, Houston J. Math., 35 (2009), 775-786. MR2534280(2010i:30048)

[2] A. Biancofiore and W. Stoll, Another proof of the lemma of the logarithmic derivative in several complex variables, Ann. of Math. Studies, 100 (1981), 29-45. MR627748 (83i:32007)

[3] G. Dethloff and T. V. Tan, A second main theorem for moving hypersurfaces targets, Houston J. Math., 37 (2011), 79-111. MR2786547

[4] H. Fujimoto, On the Gauss map of a complete minimal surface in $R^{m}$, J. Math. Soc. Japan, 35 (1983), No.2, 279-288. MR692327 (84g:53015) 
[5] H. Fujimoto, Value distribution of the Gauss maps of complete minimal surfaces in $R^{n}, J$. Math. Soc. Japan, 35 (1983), 663-681. MR714468 (85c:53011)

[6] H. Fujimoto, Nonintegrated defect relation for meromorphic maps of complete Kähler manifolds into $P^{N_{1}}(C) \times \cdots \times P^{N_{k}}(C)$, Japan. J. Math., 11 (1985), No 2, 233-264. MR884636 (88m:32049)

[7] W.K. Hayman, Meromorphic functions, Oxford Mathematical Monographs, Clarendon Press, 1964. MR0164038(29:1337)

[8] L. Karp, Subharmonic functions on real and complex manifolds, Math. Z., 179 (1982), 535554. MR652859 (84d:53042)

[9] Y. Liu and M. Ru, A defect relation for meromorphic maps on parabolic manifolds intersecting hypersurfaces, Illinois J. Math., 49 (2005), no. 1, 237-257. MR2157377 (2006c:32014)

[10] M. Ru, A defect relation for holomorphic curves intersecting hypersurfaces, American Journal of Mathematics 126 (2004), 215-226. MR2033568 (2004k:32026)

[11] M. Ru, Holomorphic curves into algebraic varieties, Ann. of Math. (2), 169 (2009), no.1, 255-267. MR2480605 (2010d:32009)

[12] W. Stoll, Introduction to value distribution theory of meromorphic maps, Lecture Notes in Math. 950 (1982), 210-359. MR672787(84a:32041)

[13] S.-T. Yau, Some function-theoretic properties of complete Riemannian manifolds and their applications to geometry, Indiana U. Math. J. 25 (1976), 659-670. MR0417452 (54:5502)

[14] A. Vitter, The lemma of the logarithmic derivative in several variables, Duke Math. J., 44(1977), 89-104. MR0432924(55:5903)

Department of Mathematics, University of Houston, Houston, Texas 77204

E-mail address: minru@math.uh.edu

Department of Mathematics, University of Houston, Houston, Texas 77204

E-mail address: ssogome@math.uh.edu 